\title{
Numerical model for the transport and degradation of pollutants through wetlands
}

\begin{abstract}
Constructed wetlands are increasingly being designed and used to treat wastewaters. The majority of constructed wetlands are designed based on steady-state releases of pollutant loading. However, in some cases (i.e., aquaculture ponds) pollutant loadings are not steadystate, rather are intermittent. Analysis based on steady-state release (inflow) will be quite different from an analysis based on intermittent loading/inflow. A simple numerical model was developed based on conservation of mass principle for the pollutants and convection through a wetland, considering a series of tanks. Tank-in-series approach assumes that the wetland is comprised of several interconnected tanks, each of which can be modelled as a continuous flow stirred tank reactor. The developed numerical model can simulate pollutant transport and degradations for steady-state, continuous and/or irregular/intermittent pollutant loadings. Numerical model results were verified with earlier developed analytical solutions for intermittent pollutant loadings. Numerical model results are very close to the results derived from analytical solutions for the same condition. The developed numerical model was used to present different scenarios using different flow rates, pond volumes, degradation constants and different masses of intermittent pollutants.
\end{abstract}

Keyword: Intermittent loading; Tanks-in-series; Constructed wetlands; Numerical modelling; Pollutant transport; Pollutant degradation; Water pollution; Wastewater treatment; Aquaculture ponds; Continuous flow stirred tank reactors; CSTR; Flow rates; Pond volumes; Degradation constants 